\title{
ChemComm
}

\section{Catalytic liquid marbles: Ag nanowire-based miniature reactors for highly efficient degradation of methylene blue $\dagger$}

Cite this: Chem. Commun., 2014, 50, 5923

Received 16th March 2014, Accepted 14th April 2014

DOI: $10.1039 / c 4 c c 01949 k$

www.rsc.org/chemcomm

\author{
Yue-E Miao, ${ }^{\text {ab }}$ Hiang Kwee Lee, ${ }^{\text {ac }}$ Wee Shern Chew, ${ }^{a}$ In Yee Phang, ${ }^{c}$ Tianxi Liu*b \\ and Xing Yi Ling*a
}

\begin{abstract}
Ag nanowire-based catalytic liquid marbles are fabricated as miniature reactors, which demonstrate highly efficient, supportfree and rate-controllable heterogeneous degradation of methylene blue, with catalytic efficiency close to $100 \%$. Our miniature catalytic liquid marbles are essential for reactions involving highly toxic/ hazardous or costly reactants, where small volume preliminary reactions are preferred.
\end{abstract}

As a new approach to isolate and manipulate liquid, ${ }^{1}$ liquid marbles have attracted increasing attention for the study of their formation, properties and potential application. ${ }^{2}$ Liquid marbles is generally spherical structures formed by rolling sessile liquid droplets over a bed of hydrophobic micro- or nano-sized particles to form a loosely packed and porous encapsulating shell at the liquid-gas interface. ${ }^{3}$ This hydrophobic shell imparts liquid marbles non-wetting properties that allow them to move easily on both solid and liquid platforms. ${ }^{4}$ Liquid marbles also demonstrate excellent mechanical stability imparted by hydrophobic powder attraction forces which maintain their structural integrity and flexibility, even when subjected to mechanical impact and deformation. ${ }^{5}$ Thus, liquid marbles can be easily manipulated by external forces, such as gravity, ${ }^{6}$ electrical, ${ }^{7}$ and magnetic field without rupturing. ${ }^{8}$ In addition, unique properties can be installed into liquid marbles via the choice of encapsulating solids, making liquid marbles highly versatile platforms for a wide range of applications, such as chemical sensors, ${ }^{4,9}$ micro-pumps, ${ }^{10}$ and miniature reactors. ${ }^{11}$

The application of liquid marbles as miniature reactors has been extensively explored due to the reduced use of chemical

\footnotetext{
${ }^{a}$ Division of Chemistry and Biological Chemistry, School of Physical and Mathematical Sciences, Nanyang Technological University, Singapore 637371. E-mail: xyling@ntu.edu.sg

${ }^{b}$ State Key Laboratory of Molecular Engineering of Polymers, Department of Macromolecular Science, Fudan University, Shanghai 200433, P. R. China.E-mail: txliu@fudan.edu.cn

${ }^{c}$ Institute of Materials Research and Engineering, $A{ }^{*}$ STAR (Agency for Science, Technology and Research), 3 Research Link, Singapore 117602

$\dagger$ Electronic supplementary information (ESI) available: Experimental details, SEM images and UV spectra of prepared samples. See DOI: $10.1039 / \mathrm{c} 4 \mathrm{cc01949k}$
}

reagents and solvents, a much confined micro-environment and precisely controllable reaction conditions. These unique properties render liquid marbles useful for minimizing the usage of highly toxic, reactive, and/or costly reagents in hazardous reactions and also for fine analyses in chemical and biological processes. ${ }^{12}$ For instance, Xue et $a l^{11 a}$ showed that liquid marbles coated with fluorinated decyl polyhedral oligomeric silsesquioxane and magnetic $\mathrm{Fe}_{3} \mathrm{O}_{4}$ powder can be used as remotely-controllable chemical miniature reactors. However, current applications of liquid marbles as miniature reactors are limited because the encapsulating shells only act as inert isolating layers to provide a confined environment and do not participate in the reactions. By using solid catalysts and/or reagents as the encapsulating layers, liquid marbles can further enforce their suitability as miniature reactors, especially in the field of heterogeneous catalysis/chemical reactions.

Metal nanoparticles are promising candidates as the building blocks for the new generation liquid marble miniature reactors due to their superior catalytic ability for a wide variety of organic and inorganic reactions. ${ }^{13}$ However, the direct use of free metal nanoparticles in heterogeneous catalysis is limited because they are susceptible to aggregation which reduces their effective catalytic surface area. Moreover, metal nanoparticles freely dispersed in liquid media generally suffer from poor recovery and reusability, severely limiting their catalytic efficiency for multiple reactions. Existing strategies to enhance the efficiency and recyclability of catalysts in heterogeneous chemical reactions generally involved the immobilization of metal nanoparticles onto various solid supports such as carbonaceous materials, ${ }^{14}$ metal oxides, ${ }^{15}$ and polymeric materials, ${ }^{16}$ and also formation of hierarchical metal nanostructures like hollow spheres or "yolk-shell" clusters. ${ }^{17}$ However, these fabrication processes involve complex protocols and harsh conditions, such as hydrothermal treatment and strong acid etching, which make the construction of selfstanding macrostructures of metal nanoparticles with high catalytic efficiency, easy manipulation and recovery still challenging. With the incorporation of solid catalysts and/or reagents as the encapsulating layers, the application of liquid marbles as a catalytic platform therefore serves as an imperative 


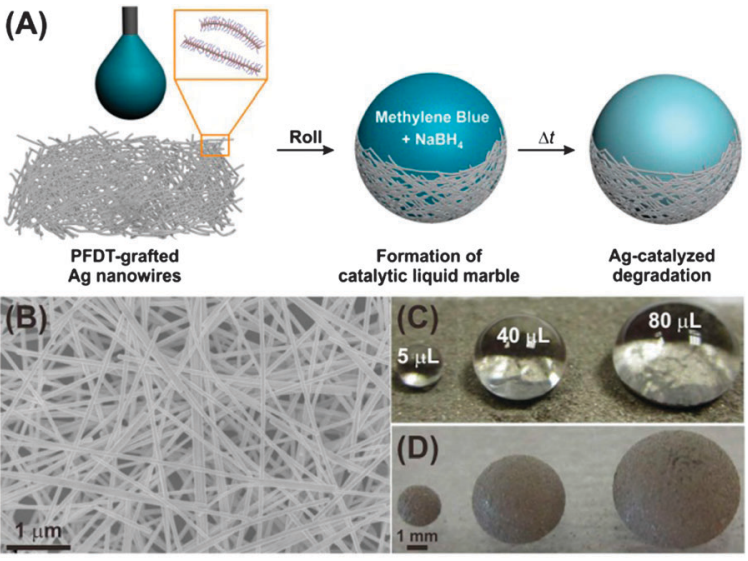

(E)

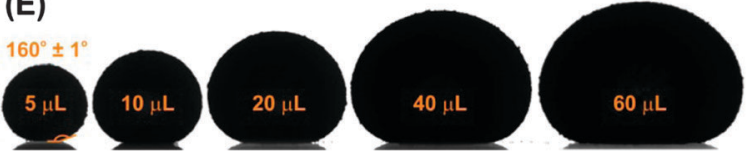

Fig. 1 (A) Schematic illustration of the preparation of catalytic liquid marbles. (B) SEM image of Ag nanowires. Digital images of 5, 40 and $80 \mu \mathrm{L}$ of (C) water droplets deposited on the bed of perfluorodecanethiolgrafted Ag nanowires and (D) catalytic liquid marbles. (E) Static contact angles of Ag nanowire-based catalytic liquid marbles fabricated using water volumes ranging from 5 to $60 \mu \mathrm{L}$.

approach in heterogeneous catalysis that allows both high catalytic efficiency and a simple yet efficient recovery protocol.

Here, we demonstrate the fabrication of $\mathrm{Ag}$ nanowire-based liquid marbles as support-free catalytic miniature reactors and their application in heterogeneous Ag-catalyzed degradation of methylene blue (Fig. 1A). Our strategy combines the superior catalytic properties of $\mathrm{Ag}$, large surface area-to-volume ratios of nanowires, and their ability to yield mechanically stable network-like porous liquid marble shells to achieve efficient catalytic reactions. Ag nanowire-based liquid marbles of various sizes are fabricated using sessile water droplets ranging from 5 to $80 \mu \mathrm{L}$ and their catalytic activities are quantitatively monitored using a UV-vis spectrometer. We then systematically demonstrate the kinetic modulation of Ag-catalyzed reactions and investigate the recyclability and reusability of the $\mathrm{Ag}$ nanowire catalyst.

Monodispersed Ag nanowires are synthesized in high yield using a modified polyol process, ${ }^{18}$ with an average length over $10 \mu \mathrm{m}$ and an average diameter of $65 \pm 14 \mathrm{~nm}$ (Fig. 1B).

To render hydrophobicity essential for supporting a spherical sessile droplet of water, $1 H, 1 H, 2 H, 2 H$-perfluorodecanethiol is grafted onto the as-prepared $\mathrm{Ag}$ nanowires via ligand exchange. No obvious change in the morphology of perfluorodecanethiol-grafted $\mathrm{Ag}$ nanowires is observed (Fig. S1A, ESI $\dagger$ ). The observation is further supported by the negligible change in extinction spectra after the ligand exchange process (Fig. S1B, ESI $\dagger$ ), where the characteristic transverse plasmon peaks at $383 \mathrm{~nm}$ and $349 \mathrm{~nm}$ remain unchanged. These transverse surface plasmon peaks correspond to the out-of-plane quadrupole resonances of $\mathrm{Ag}$ nanowires with pentagonal cross-section. ${ }^{19}$ When different sessile water droplets of volume ranging from 5 to $80 \mu \mathrm{L}$ are dispensed on the bed of perfluorodecanethiol-grafted Ag nanowires, spherical water droplets are formed (Fig. 1C). This clearly illustrates the superior anti-wetting properties of the perfluorodecanethiol-grafted $\mathrm{Ag}$ nanowires, which are essential for subsequent catalytic liquid marble formation.

Catalytic liquid marbles are formed by rolling sessile water droplets over the hydrophobic perfluorodecanethiol-grafted $\mathrm{Ag}$ nanowires. The Ag nanowires are observed to assemble at the water/air interface spontaneously (Fig. 1D) and the resulting encapsulating shell is determined as a free-standing microstructure made up of loosely-packed Ag nanowires (Fig. S2, ESI $\dagger$ ). The length of pulverized $\mathrm{Ag}$ nanowires used for the formation of catalytic liquid marbles is also observed to be shorter compared to the unpulverized Ag nanowires. Nevertheless, pulverization of $\mathrm{Ag}$ nanowire powder is essential for the formation of uniform catalytic liquid marbles. These shorter Ag nanowires are able to provide a porous Ag nanowire network with potentially high catalytic surface area.

Minimal interaction between catalytic liquid marbles and the underlying solid platform is also demonstrated by measurement of their contact angles. Static contact angles over $160^{\circ}$ are generally observed for all catalytic liquid marbles, ranging from 5 to $60 \mu \mathrm{L}$, when deposited on a hydrophilic substrate (Fig. 1E). The high contact angle $\left(\geq 150^{\circ}\right)$ of the liquid marble is another indication of the non-wetting properties of $\mathrm{Ag}$ nanowires, which prevent the direct interaction of encapsulated water with the underlying platform. Although slight shape distortions from spherical structures are observed for liquid marbles with volume larger than $5 \mu \mathrm{L}$ as a consequence of gravity, the intact, isolated and flexible puddle-like structures are still able to act as miniature reactors. Moreover, the result clearly demonstrates that the reaction capacities of liquid marble-based miniature reactors can be systematically modulated by accurately controlling the volume of water droplets used for the formation of liquid marbles. Hence, the versatility of liquid marble-based miniature reactors enables them to potentially accommodate a wide range of volumes of reaction mixtures, ranging from $5 \mu \mathrm{L}$ to $60 \mu \mathrm{L}$.

To investigate the performance of Ag nanowire-based liquid marbles as catalytic miniature reactors, the catalytic reduction of encapsulated aqueous methylene blue in the presence of sodium borohydride $\left(\mathrm{NaBH}_{4}\right)$ is studied. ${ }^{20}$ Typically, $5 \mu \mathrm{L}$ of catalytic liquid marbles containing $2 \mathrm{mM}$ methylene blue solution and $0.2 \mathrm{M}$ $\mathrm{NaBH}_{4}$ are prepared. The catalytic liquid marbles are then ruptured at pre-defined timings and the encapsulated aqueous methylene blue solutions are collected, diluted 100-fold, where the color intensity of the solution is first examined. Using $5 \mu \mathrm{L}$ of $\mathrm{Ag}$ nanowire-based liquid marbles, the intensity of the blue coloration of methylene blue solutions is visibly reduced within the second min and eventually turns colorless by the tenth min (Fig. 2A). In contrast, no apparent color change is noted in the methylene blue solution in the absence of the encapsulating $\mathrm{Ag}$ nanowire shell (Fig. 2B).

The extinction of the reacting aqueous solution is then monitored over a duration of $10 \mathrm{~min}$ to quantitatively examine the degree of methylene blue degradation (Fig. 2C). Using the characteristic $665 \mathrm{~nm}$ absorption band of the methylene blue, a calibration plot relating methylene blue concentration and 

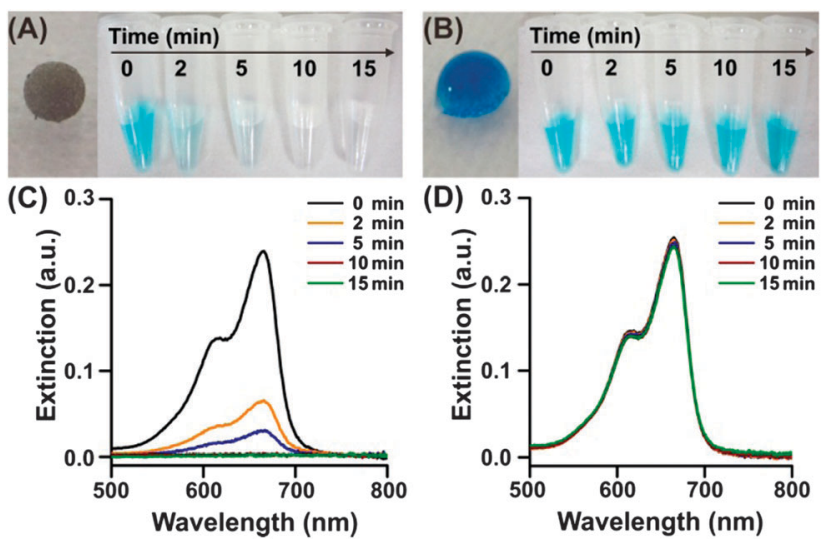

Fig. 2 Digital images depicting the color change of aqueous methylene blue solutions (A) in Ag nanowire-based liquid marbles and (B) without forming liquid marbles, at different time intervals. All setups use $5 \mu \mathrm{L}$ of methylene blue solution. ( $C$ and D) UV-vis spectra of methylene blue solutions measured at different time points of (A) and (B), respectively.

extinction is obtained (Fig. S3, ESI $\dagger$ ). Hence, the concentration of methylene blue at selected time can be easily interpolated based on the calibration plot. The methylene blue degradation rate is characterized by plotting $C / C_{0}$ against time, where $C_{0}$ and $C$ represent methylene blue at its initial concentration and its concentration at time $t$, respectively (Fig. 3A). The extinctionduration plot depicts an exponential decrease in extinction with time for the $5 \mu \mathrm{L} \mathrm{Ag}$ nanowire-based catalytic liquid marble, which indicates drastic degradation of methylene blue to nonabsorbing products. ${ }^{21}$ Within 2 min of the reaction time, $\geq 75 \%$ of the initial methylene blue molecules are degraded $\left(C / C_{0}=\right.$ 0.25 ) and a further decrease in methylene blue concentration is observed as the reaction proceeds. At $t \geq 10 \mathrm{~min}$, the reduction efficiency of methylene blue reaches nearly $100 \%\left(C / C_{0}=0.02\right)$,
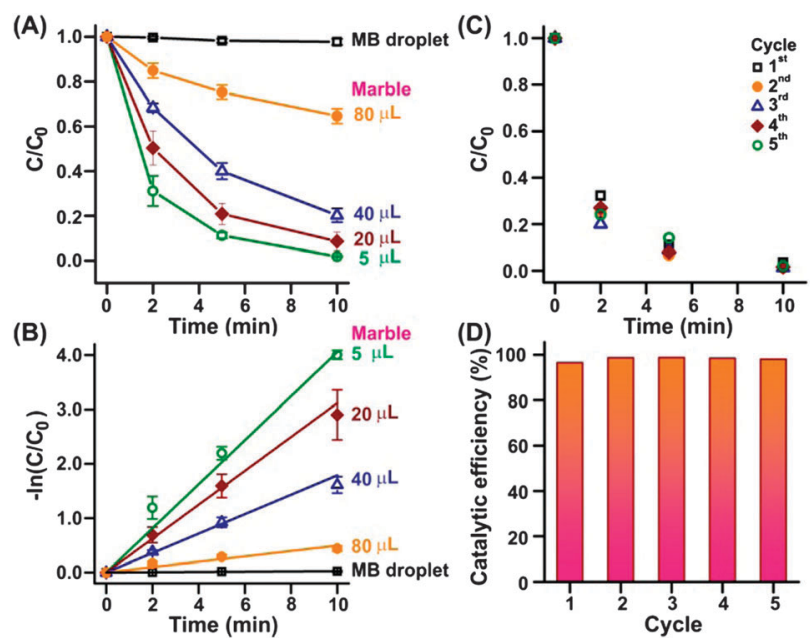

Fig. 3 Plots of $(A)$ normalized methylene blue concentrations $\left(C / C_{0}\right)$ and (B) $-\ln \left(C / C_{0}\right)$ versus time using catalytic $\mathrm{Ag}$ nanowire liquid marbles of different sizes. (C) Correlation of methylene blue concentration with time using $5 \mu \mathrm{L} \mathrm{Ag} \mathrm{nanowire-based} \mathrm{catalytic} \mathrm{liquid} \mathrm{marbles} \mathrm{for} 5$ degradation cycles. (D) Comparison of catalytic efficiency and reusability of Ag nanowires over 5 degradation cycles. indicating that almost all methylene blue molecules have been degraded. The rapid degradation of methylene blue using Ag nanowire-based catalytic liquid marbles can be attributed to two reasons. Firstly, the presence of Ag serves as an efficient electron relay between nucleophilic $\mathrm{NaBH}_{4}$ and electrophilic methylene blue for the catalytic reduction-degradation process. ${ }^{21}$ Secondly, the high surface area-to-volume ratio imparted by $\mathrm{Ag}$ nanowires and the aforementioned porous network structure allow the exposure of large amounts of catalytic sites for efficient degradation reaction. The need for porous network structure for efficient catalysis is further confirmed using compacted-shell $\mathrm{Ag}$ nanocube-based liquid marbles $(5 \mu \mathrm{L})$, which provide a catalytic efficiency of only $49 \%$ after 10 min reaction time (Fig. S4, $\mathrm{ESI} \dagger$ ), approximately half the catalytic efficiency of $\mathrm{Ag}$ nanowire-based liquid marbles (refer to the ESI $\uparrow$ for detailed discussion).

We further illustrate the necessity of the Ag catalyst by comparing with an identical experimental set-up but in the absence of the encapsulating Ag layer. The non-encapsulated methylene blue solution exhibits no obvious degradation of methylene blue by $\mathrm{NaBH}_{4}$ from its appearance and UV-vis spectra (Fig. 2B and D), with $C / C_{0}$ approximating 1 even after $10 \mathrm{~min}$. Hence, it is evident that the heterogeneous $\mathrm{Ag}$ nanoparticle-based catalyst is essential for the degradation reaction and can be easily incorporated into the reacting mixtures in the form of liquid marbles without the need for supporting platforms. Although the catalytic efficiency of liquid marbles may be lower compared to an equal volume $(5 \mu \mathrm{L})$ of aqueous methylene blue dispersed with an approximately equal amount of Ag nanowires (Fig. S5, ESI $\dagger$ ), we emphasize that such dispersion cannot act as an isolated robust miniature reactor and the $\mathrm{Ag}$ catalyst cannot be recovered easily in comparison to the use of liquid marbles.

The chemical kinetics of our catalytic liquid marbles can be easily modulated by systematically varying their sizes using different volumes of aqueous reaction solution (5, 20, 40 and $80 \mu \mathrm{L})$. By monitoring the intensity of the $655 \mathrm{~nm}$ extinction peak over time (Fig. 3A and Fig. S6, ESI $\dagger$ ), we observe a general exponential decrease of $C / C_{0}$ as the reaction proceeds for all reaction volumes. Hence, we assume the catalytic reaction as a pseudo-first order reaction where the first-order reaction kinetics equation can be applied, i.e. $\ln \left(C_{0} / C\right)=k_{\text {app }} \times t$, where $k_{\text {app }}$ is the degradation rate constant (Fig. 3B). ${ }^{17 b}$ The apparent rate constant $k_{\text {app }}$ values are $0.41,0.31,0.18$ and $0.05 \mathrm{~min}^{-1}$ for $5,20,40$ and $80 \mu \mathrm{L}$ liquid marbles, respectively. The results clearly indicate that the kinetic rate of the catalytic reactions can be easily manipulated simply by controlling the size of the liquid marble. An increase in the liquid marble size leads to a significant decrease of the catalytic reaction rate. Although the reaction rate of catalytic liquid marbles of $<5 \mu \mathrm{L}$ is expected to be faster, it is technically challenging to obtain accurate sessile droplets of $<5 \mu \mathrm{L}$ using a pipette/manual dispenser. Moreover, at volumes $<5 \mu \mathrm{L}$, it is difficult to visibly differentiate between a liquid marble from its ruptured state.

Considering that temperature, pressure and initial reactant concentration are kept constant throughout the reactions, the decreasing rate constant $k_{\text {app }}$ with respect to the increase in the volume/size of liquid marbles can be attributed to a decrease in 
the efficiency of mass transfer of reactants to the catalytic active sites at the surface, which is a major factor affecting the kinetics of a heterogeneous catalytic reaction. In addition, the increase of the analyte-to-surface area ratio with an increasing volume of reaction solution can also contribute to a longer degradation time of methylene blue and a slower rate constant (or reaction kinetics) (see the ESI $\dagger$ for more discussion on volume-controlled reaction kinetics). Therefore, the catalytic liquid marbles have clearly demonstrated their ability to modulate chemical kinetics just simply by changing their size, which can be potentially applied as rate-controllable catalytic miniature reactors.

We also examine the recyclability and reusability of perfluorodecanethiol-grafted Ag nanowires by measuring their catalytic performance for multiple degradation cycles. The Ag nanowire powder on catalytic liquid marbles can be easily recycled by removing the interior aqueous solution and re-rolling new droplets of methylene blue solution on the powder bed to form catalytic liquid marbles without additional treatment. We observe that the morphology of the recycled Ag nanowire powder (Fig. S7, $\mathrm{ESI} \dagger$ ) exhibits negligible wear and tear, which is essential for the maintenance of the integrity of the porous network liquid marble shell during the recycle test. Using the recycled Ag nanowires for the subsequent formation of catalytic liquid marbles, catalytic efficiencies of $>95 \%$ towards the degradation of methylene blue are still consistently obtained even after five reaction cycles (Fig. 3C and D). Hence, the catalytic liquid marble has been demonstrated to be a promising cost-effective, highly efficient miniature reactor with rate-modulating capability and long cycle life without losing its catalytic efficiency.

In summary, Ag nanoparticle-based catalytic liquid marbles have been fabricated as support-free, rate-controllable catalytic miniature reactors with high methylene blue reduction efficiency close to $100 \%$ and excellent reusability over 5 catalytic cycles. The ensemble of benefits therefore enables the use of catalytic liquid marbles as attractive alternative miniature reactors, which are highly appealing for support-free heterogeneous catalysis of small volume reactions. This is especially important in fields involving highly toxic and costly reactants and/or hazardous reactions where preliminary reactions involving small volume are preferred. Our protocol for the fabrication of catalytic liquid marbles is also generic and can be extended to micro- and nanoparticles of different morphology and materials to tailor their reactivity for a wide range of chemical reactions.
X.Y.L. is thankful for the support from National Research Foundation, Singapore (NRF-NRFF2012-04), and the Nanyang Technological University's start-up grant. T.L. and Y.M. are grateful for the financial support from the National Natural Science Foundation of China (51125011). H.K.L. is thankful for the $A^{*}$ STAR Graduate Scholarship support from $A^{*}$ STAR, Singapore.

\section{Notes and references}

1 P. Aussillous and D. Quere, Nature, 2001, 411, 924.

2 (a) E. Bormashenko, Y. Bormashenko, A. Musin and Z. Barkay, ChemPhysChem, 2009, 10, 654; (b) E. Bormashenko, Y. Bormashenko and A. Musin, J. Colloid Interface Sci., 2009, 333, 419.

3 P. Aussillous and D. Quéré, Proc. R. Soc. A, 2006, 462, 973.

4 J. Tian, T. Arbatan, X. Li and W. Shen, Chem. Eng. J., 2010, 165, 347.

5 L. Zhang, D. Cha and P. Wang, Adv. Mater., 2012, 24, 4756.

6 D. Zang, Z. Chen, Y. Zhang, K. Lin, X. Geng and B. P. Binks, Soft Matter, 2013, 9, 5067.

7 S.-Y. Tang, V. Sivan, K. Khoshmanesh, A. P. O’Mullane, X. Tang, B. Gol, N. Eshtiaghi, F. Lieder, P. Petersen, A. Mitchell and K. Kalantar-zadeh, Nanoscale, 2013, 5, 5949.

8 Y. Zhao, J. Fang, H. Wang, X. Wang and T. Lin, Adv. Mater., 2010, 22, 707.

9 H. K. Lee, Y. H. Lee, I. Y. Phang, J. Wei, Y.-E. Miao, T. Liu and X. Y. Ling, Angew. Chem., 2014, DOI: 10.1002/anie.201401026.

10 E. Bormashenko, R. Pogreb, Y. Bormashenko, A. Musin and T. Stein, Langmuir, 2008, 24, 12119.

11 (a) Y. Xue, H. Wang, Y. Zhao, L. Dai, L. Feng, X. Wang and T. Lin, Adv. Mater., 2010, 22, 4814; (b) T. Arbatan, L. Li, J. Tian and W. Shen, Adv. Healthcare Mater., 2012, 1, 80; (c) J. Tian, N. Fu, X. D. Chen and W. Shen, Colloids Surf., B, 2013, 106, 187.

12 (a) K. Jensen, Nature, 1998, 393, 735; (b) S.-Y. Teh, R. Lin, L.-H. Hung and A. P. Lee, Lab Chip, 2008, 8, 198.

13 J.-Z. Guo, H. Cui, W. Zhou and W. Wang, J. Photochem. Photobiol., A, 2008, 193, 89.

14 B. Hu, Y. Zhao, H.-Z. Zhu and S.-H. Yu, ACS Nano, 2011, 5, 3166.

15 N. Zheng and G. D. Stucky, J. Am. Chem. Soc., 2006, 128, 14278.

16 (a) H.-L. Jiang and Q. Xu, Chem. Commun., 2011, 47, 3351; (b) D. Hu, Y. Huang, H. Liu, H. Wang, S. Wang, M. Shen, M. Zhu and X. Shi, J. Mater. Chem. A, 2014, 2, 2323; (c) Y. Huang, H. Ma, S. Wang, M. Shen, R. Guo, X. Cao, M. Zhu and X. Shi, ACS Appl. Mater. Interfaces, 2012, 4, 3054; (d) X. Fang, H. Ma, S. Xiao, M. Shen, R. Guo, X. Cao and X. Shi, J. Mater. Chem., 2011, 21, 4493.

17 (a) M. Xiao, C. Zhao, H. Chen, B. Yang and J. Wang, Adv. Funct. Mater., 2012, 22, 4526; (b) S. Shi, M. Wang, C. Chen, J. Gao, H. Ma, J. Ma and J. Xu, Chem. Commun., 2013, 49, 9591.

18 M. Chen, I. Y. Phang, M. R. Lee, J. K. W. Yang and X. Y. Ling, Langmuir, 2013, 29, 7061.

19 M. S. Goh, Y. H. Lee, S. Pedireddy, I. Y. Phang, W. W. Tjiu, J. M. R. Tan and X. Y. Ling, Langmuir, 2012, 28, 14441.

20 (a) S. Sohrabnezhad, A. Pourahmad, R. Rakhshaee, A. Radaee and S. Heidarian, Superlattices Microstruct., 2010, 47, 411; (b) N. Gupta, H. P. Singh and R. K. Sharma, J. Mol. Catal. A: Chem., 2011, 335, 248. 21 N. R. Jana, T. K. Sau and T. Pal, J. Phys. Chem. B, 1998, 103, 115. 